\title{
An Intelligent System in Healthcare using Fuzzy Measures
}

\author{
Amol S. Wagholikar and Jun Jo \\ School of Communication and Information Technology \\ Griffith University \\ Gold Coast, Queensland, Australia \\ \{a.wagholikar,j.jo\}@griffith.edu.au
}

\begin{abstract}
Aggregation plays a central role in human thought. There is a need to develop better data aggregation mechanisms in various real-life applications involving intelligent systems. Application of fuzzy measures and fuzzy integrals is a significant development in this pursuit. However, there are certain issues associated with practical implementation of fuzzy measures. This work proposes an approach to resolve those issues using similarity-based reasoning. Application of fuzzy measures in healthcare applications is an interesting development in the area of intelligent systems. This paper also discusses application of fuzzy measures in modern health care applications using the proposed approach. This work demonstrates the suitability of fuzzy measures in critical health care systems.
\end{abstract}

\section{INTRODUCTION}

A decision-making problem relies on data aggregation tools in order to evaluate given alternatives against given set of criteria. There are various decision-making models. Multicriteria decision-making is a systematic and formal decision model [1]. It is one of the common models used in solving decision problems involving interactive criteria. Most of the real-life decision problems are characterized by the presence of interactive criteria. The interaction between the criteria must be considered to provide intelligent decision support. Traditional additive measures such as weighted average are inadequate to model the interaction between the criteria [2]. This calls for novel approaches that can address this issue. Non-additive measures such as fuzzy measures are capable of modeling the interaction between the criteria. However, there are certain issues in transferring the theoretical ability in practical situations. This is mainly due the complexity in determining fuzzy measure coefficients in practical applications. It is always important to determine practical ways of applying fuzzy measures in real-life applications [3]. In this pursuit, the main objective of this work is to investigate the issue of fuzzy measure acquisition 978-1-4244-2197-8/08/\$25.00 @2008 IEEE and develop a methodology to resolve the issue in practical problems. This work demonstrates the methodology in an intelligent system designed to predict heart rate of the patients. This system involves various interactive parameters same as a MCDM problem mentioned earlier. It is important to model the interaction between the parameters. Fuzzy measures are good tools in such application.

The proposed approach rests on the conceptual foundation of similarity-based reasoning [4]. There are various approaches suggested for resolving the issue of fuzzy measure acquisition [5][6]. These approaches mainly suggest the use of past precedents for determination of fuzzy measures. This work has extended this notion by applying similarity-based reasoning for resolving this issue. This work illustrates a significant application of similarity-based reasoning especially case-based reasoning for application of fuzzy measures in intelligent systems. This research contributes to the theory of fuzzy measures and similarity-based reasoning with a practical application of intelligent systems in health care. Such combination of important theories illustrates the significance of this research. This work offers a new perspective towards the application of fuzzy measures in real-life applications.

\section{About Non-AdDitive Measures}

We are addressing the issue of fuzzy measure determination in the context of decision-making problem and data aggregation. MCDM is a systematic and formal decision model. It consists of evaluation of given set of alternatives against a given set of criteria. The chosen criteria can be dependent or independent. They can be positively or negatively related with each other [7]. The interaction between the criteria should be considered while aggregating the criteria evaluations. Normally, additive measures such as weighted average are used for aggregation. Due to the additive nature of these measures, these measures are inadequate to model the phenomenon of interaction.

\section{A. Non-additive measures}


Non-additive measures such as fuzzy measures and fuzzy integrals have the ability to model the issue of dependence between the criteria.

\section{B. Fuzzy Measures}

Fuzzy measures model the combined importance of a sub-set of criteria. These are monotone set functions defined over a universal set $\mathrm{C}$ and a non-empty family $\rho$ of sub-sets (i.e. power set) of $\mathrm{C}$. It is defined as $\mu: \rho \rightarrow[0,1]$, where $\mu(\phi)=0 \& \mu(X)=1$. The fuzzy measures possess the property of super additivity and sub additivity, which models the positive and negative type of interaction respectively. For more detailed description of these properties, readers are kindly referred to the literature [8].

\section{Fuzzy Integrals}

Fuzzy integrals are the incremental summation of the product of the criteria evaluation and its fuzzy measures. There are two types of commonly used fuzzy integrals viz Choquet integral and Sugeno integral [9]. Choquet integral is used for a quantitative setting whereas Sugeno integral is proposed for a qualitative setting. Since we have assumed a quantitative setting, we would use Choquet integral for this work.

Let $C=\left\{c_{1}, c_{2}, \ldots ., c_{n}\right\}$ be the set of elements. $\mu$ be the fuzzy measure on $\mathrm{C}$. The elements of $\mathrm{C}$ can be criteria in MCDM, players in cooperative games or set of beliefs in Demestershafer theory. The Choquet integral of the function $f: \mathrm{C} \rightarrow$ $[0,1]$ with respect to $\mu$ is given by.

$$
\mathrm{Ch}_{\mu}=\sum_{\mathrm{i}=1}^{\mathrm{n}}\{\mathrm{f}(\mathrm{c}(\mathrm{i}))-f(\mathrm{c}(\mathrm{i}-1))\} \mu(\mathrm{A}(\mathrm{i}))
$$

Where (i) indicates that the indices have been permuted such that -

$0 \leq f(\mathrm{c}(1)) \leq f(\mathrm{c}(2)) \leq \ldots \ldots . . \leq f(\mathrm{c}(\mathrm{n})) \leq 1$ and $\mathrm{A}(\mathrm{i})=\{\mathrm{c}$ (i) $, \ldots \ldots \ldots \ldots, \mathrm{c}(\mathrm{n})\}$. We can observe that for aggregating " $n$ " elements, we need to determine $2^{n}-1$ fuzzy measure coefficients. Hence their practical determination is the main issue especially for higher values of " $n$ ".

\section{DETERMINATION OF FUZZY MEASURES}

As mentioned earlier, fuzzy measure is the coefficient of the power set of sub-sets of criteria. The issue of determining the fuzzy measures is mainly marked by the complexity due to high number of fuzzy measure coefficients. For e.g. when $n$ $=3$, we can have 7 coefficients including individual as well as sub-sets of criteria. The number of fuzzy measure coefficients can be very large for higher values of $n$, say when $n$ is equal to 7. To resolve this issue certain data-driven methods [10], interactive methods [11] have been suggested. Also some approaches suggest the use of pre-defined fuzzy measures to determine the new fuzzy measures or use of global evaluations from the training data [12]. We are extending this notion of using training data about the past decisions. We are proposing a new method where the training data is in the form of a casebase. The case-base will consist of data about the past decisions made under MCDM setting.

\section{A. Proposed Approach}

We are extending the notion of using learning data about the past decisions. Reasoning by analogy is the main theoretical foundation of our approach [13]. New problems can be solved using solutions of the past similar problems. We are proposing a new method where the learning data is in the form of a casebase. Case-based reasoning (CBR) allows solving new problems based on the solutions from the past similar problems. The undertaken issue of fuzzy measure determination focuses on modeling the human decision making processes. Hence CBR can be a good tool for this purpose.

B. Proposed CBR-based Approach

We are proposing the following model.

1. Let $C=\left\{C_{1}, C_{2}, \ldots \ldots . . C_{n}\right\}$ be the set of criteria against which a set $\mathrm{A}$ of alternatives $\left\{A_{1}, A_{2}, \ldots \ldots \ldots, A_{m}\right\}$ is to be evaluated. The individual weights, $\mathrm{w}_{\mathrm{i}}$ on the criteria is elicited from the decision maker such that $\sum_{i=1}^{n} w_{i}=1$. For this above decision problem, we have case-base in the following form.

i. A set of attributes same as the new decision problem or target problem mentioned above.

ii. Absolute values of each attribute.

iii. A set of individual weights defined by the decision maker at the previous decision making instance.

iv. A set $S$ of fuzzy measure coefficients defined previously. We assume that these coefficients are constructed using expert's feedback.

v. Global evaluation of the object obtained by using the Choquet integral and the fuzzy measure coefficients obtained from the experts.

2. After constructing the above data set, the similarity of the cases w.r.t to the new decision problem is determined using absolute value-based City-block distance metric or Manhattan distance metric [14]. The Manhattan distance metric is defined as the absolute difference between the values of pair of attributes or criteria I.e.

$$
d_{i j}=\sum_{k=1}^{n}\left|x_{i k}-x j k\right|
$$

Where $x_{i} \& x_{j}$ are the values of the attribute " $k$ ". For computing the similarity, the distance values should be normalized in the range $[0,1]$ by the maximum distance $d_{\max }$ between the corresponding attribute values in the new problem and the case base. The global similarity of a given case will be calculated by simple weighted average of the 
local similarities of the given attributes. Thus the distance between the cases will be

$$
d\left(\text { Caset }, \text { Case }_{u}\right)=\frac{\sum_{k=1}^{n} w_{k} d_{i j}}{\sum_{k=1}^{n} w_{k}}
$$

Based on this distance, the similarity is calculated as $\operatorname{Sim}\left(\right.$ Case $_{t}$, Case $\left._{\mathcal{U}}\right)=1-d\left(\right.$ Case $_{\text {, }}$ Case $\left._{\mathcal{U}}\right)$

Where Case $_{\mathrm{t}}=$ Target case i.e. the new decision problem \& Case $_{\mathrm{u}}=$ Case from the case library i.e. past decision problems. 3. After such similarity computation the case with maximum similarity would be retrieved and the values of its fuzzy measures would be considered for computing the global evaluation of the given alternatives.

\section{FUZZY INTELLIGENT SYSTEM}

MEASURE-BASED

Healthcare applications involve parameters with varying degree of relationship between them. Fuzzy measures are good tools in such applications. We now discuss an intelligent system used for heart rate prediction. The effect of physical activity on heart rate has been studied in a number of research areas including energy expenditure measurement, autonomic nervous system assessment, sports research. However, there have been few studies on the prediction relationship between them.

Heart rate (HR) is generally measured as a series of time intervals (the so-called RR intervals) between the heart cycles that are obtained from the electrocardiogram (ECG) [15]. Analysis on HR has become a popular noninvasive tool for studies on cardiopathy and exercise physiology. One of the limitations associate with the HR monitoring technique is that it is difficult to identify whether the HR increases are due to physical activity (PA) or mental activity [16], especially when the HR increase is modest.

Figure 1 shows the block diagram of the proposed system. The left part of Figure 1 assumes the subject (user) wearing the monitor.

The middle part of Figure 1 is the preprocessor which converts the acquired variables such as ECG and acceleration signals from the monitor into a usable format. There could be different variables that can have impact on the heart rate. The outputs of the preprocessor include synchronized sequences of the input variables at interval time $n$, which are forwarded to the FFNN as inputs. The output of the neural network is HR $(n+1)$.

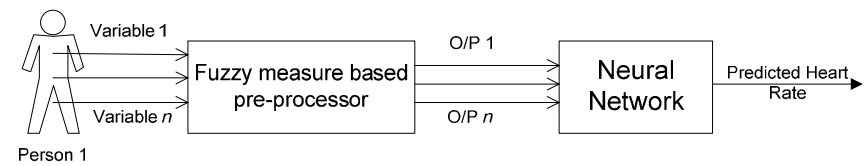

Figure 1: The block diagram of the whole system.
The important aspect of the above application is to determine the inputs to the Neural Network. These inputs will be determined by the interactive variables.

\section{A. Illustration}

The following sample data is constructed for the illustration of the proposed approach. The data set consists of a set of 4 outputs. These alternatives are evaluated against a set of 5 criteria or variables. The absolute values of the criteria for a given outputs are expressed in numeric as well as linguistic terms.

TABLE II. SAMPLE DATA SET FOR HEART RATE PREDICTION PROBLEM

\begin{tabular}{|l|l|l|l|l|l|}
\hline & ECG & $\begin{array}{l}\text { Physical } \\
\text { Activity }\end{array}$ & Age & Gender & Health Level \\
\hline Weights & 0.3 & 0.2 & 0.2 & 0.1 & 0.2 \\
\hline O/P 1 & 60 & Moderate & 26 & Female & OK \\
\hline O/P 2 & 70 & Good & 30 & Male & OK \\
\hline O/P 3 & 70 & Good & 27 & Female & Good \\
\hline O/P 4 & 70 & OK & 28 & Male & Good \\
\hline
\end{tabular}

Suppose for the above data set, we have the following casebase.

TABLE III.

CASE BASE OF PAST DECISIONS

\begin{tabular}{|l|l|l|l|l|l|}
\hline & ECG & $\begin{array}{l}\text { Physical } \\
\text { Activity }\end{array}$ & Age & Gender & $\begin{array}{l}\text { Health } \\
\text { Level }\end{array}$ \\
\hline Case 1 & 60 & Moderate & 27 & Male & Good \\
\hline Case 2 & 72 & Good & 30 & Male & Good \\
\hline Case 3 & 65 & Moderate & 26 & Female & OK \\
\hline Case 4 & 72 & Good & 28 & Male & Good \\
\hline
\end{tabular}

For the above data set, we conducted several experiments for determining similarity of the alternatives in Table II with respect to the case-base in Table III using SPSS 3.0. We conducted experiments with different similarity measures. Following table shows the results using City-block distance measures chosen for this approach.

TABLE IV.

PROXIMITY MATRIX - SPSS OUTPUT FOR CASE SIMILARITY USING CITY-BLOCK DISTANCE MEASURE

\begin{tabular}{lcccc}
\hline \multicolumn{5}{c}{ Absolute City Block Distance } \\
\hline O/P 1 & Case 1 & Case 2 & Case 3 & Case 4 \\
O/P 2 & .008 & .009 & .000 & .008 \\
O/P 3 & .035 & .017 & .027 & .035 \\
O/P 4 & .005 & .018 & .008 & .000 \\
\hline
\end{tabular}

This is a dissimilarity matrix. From this dissimilarity matrix, we can observe that $-\mathrm{O} / \mathrm{P} 1$ is similar to Case $3, \mathrm{O} / \mathrm{P} 2$ is similar to Case 2, O/P3 is similar to Case 4, O/P4 is similar to Case 4. Based on the above similarities and corresponding fuzzy 
measures of the similar cases, the global evaluations of all the variable outputs is given by the proposed case-based system using Choquet integral. These outputs were sent to the neural network. Many such experiments were performed using a monitor. Neural network was trained with sample data and pre-processor outputs were used to determine the heart rate.

The portable ECG monitor, Alive Heart Monitor (AHM), was used to monitor and record the HR and PA signals simultaneously for a period of time with relatively high accuracy.

Owing to its small size and light weight, the AHM can be worn comfortably during normal daily activities. The monitor gathers the single-channel ECG signal from a pair of electrodes attached at certain positions of the subject's skin and 3-D physical activity (acceleration) signals from a built-in 3axis-accelerometer. The collected data can be saved in an internal SD memory card or transmitted to a PC, Smartphone or PDA using Bluetooth in real-time. The AHM uses a rechargeable lithium-ion battery which provides about four days of data saving or three days of wireless-transmission, continuously.

\section{CONCLUSION}

We discussed an important issue of acquisition of fuzzy measures in practical applications. This issue was investigated in detail. In this paper, we presented an interactive model for determining non-additive measures to resolve the undertaken issue. We have proposed a model using similarity-based reasoning. We have illustrated our model with a sample data set and through an experimental implementation in a healthcare application. This model demonstrated the original, extensive and significant contribution of our research.

\section{References}

[1]. D.R. Anderson, D.J. Sweeney, T.A. Williams, An introduction to management science Quantitative approaches to decision-making, 9 th Ed., Rochester Institute of technology, 2000.

[2]. G. Choquet, Theory of capacities, Ann.Inst.Foruier 5,pp $195,1953$.

[3]. Z. Wang, G.J. Klir, Fuzzy Measure Theory, Plenum Press, New York, 1992.

[4]. Kojadinovic, I., Modelling interaction phenomena using fuzzy measures: on the notions of interaction and independence, Fuzzy sets and Systems 135 (2003)pp. 317-340.

[5]. Zeleny, M. 1982. Multiple Criteria Decision Making, McGrawHill, New York.

[6]. J. Marichal, Aggregation Operators for Multicriteria decision aid, Ph.D. thesis, University of Li'ege, Belgium, 1999.

[7]. M.Grabisch, Fuzzy integral in multi-criteria decision making", Fuzzy Sets and Systems, 69, pp.279-298,1995.

[8]. M. Sugeno, Theory of fuzzy integrals and its applications, $\mathrm{PhD}$ Dissertation, Tokyo Institute of Technology, Japan, 1974.
[9]. Klir G, J., Constructing fuzzy measures in expert systems, fuzzy sets and systems 92 (1997),pp. 251-264.

[10]. Klir, G, J., Wang, Z., Wang, W. 1996. Constructing Fuzzy Measures by Transformations, J.Fuzzy Math. 4 (1), pp.207-215.

[11]. Sun, Z \& Finnie, G 2003, Intelligent techniques in E-Commerce: A Case Based reasoning Perspective, Physica-Verlag, Berlin.

[12]. Vosniadou, S \& Ortony, A 1989, Similarity and Analogical Reasoning, Cambride University Press.

[13]. Kardi, T. (2005): Similarity Measurement.

http: \|people.revoledu.com $\backslash$ kardi $\backslash$ tutorial $\backslash$ Similarity, ViewedMarch (2005).

[14]. Bilgic, T., Turksen, I.: Measurement of Membership Functions: Theoretical and Empirical Work, Handbook of Fuzzy Sets and Systems, Vol.1, foundations, (1997).

[15]. Léger L, Thivierge M. Heart rate monitors: validity, stability and functionality. Physician Sports Med 1988; 16 (5): pp.143-51

[16]. Haskell WL, Yee MC, Evans A, Irby PJ. Simultaneous Measurement of. Heart-Rate and Body Motion to Quantitate Physical-Activity. Med Sci Sports Exercise 25: pp.109-115, 1993. 